\title{
Fluorescence microscopy gets a frequency boost
}

An imaging strategy based on telecommunications technology dramatically accelerates fluorescence microscopy.

Microscopy has made remarkable gains in terms of spatial resolution, but in many cases these advances have come at the cost of speed: smaller numbers of photons generally mean longer data collection times.

Bahram Jalali's lab at the University of California at Los Angeles has now published a potential solution that draws on a technique commonly used in wireless communication. Fluorescence imaging using radiofrequencytagged emission (FIRE) entails splitting a laser beam into multiple beams that have been shifted in angle and frequency. A device called an interferometer then combines these various beams with light from another laser; the difference in frequency between each of the combined beams yields a distinct rhythmic interference pattern, or 'beat'. This results in a 'comb' of parallel beams with distinct beat frequencies, which is used to scan the sample. Each of the resulting pixels is thus uniquely marked by being excited at a distinct frequency, which in turn makes it possible to obtain fluorescence data at diffraction-limit resolution from many pixels simultaneously. "It's the same way that a wireless Internet router works, where different computers are communicating in different channels in different frequencies," explains Eric Diebold, a postdoc in Jalali's lab.

FIRE proved capable of generating images from cultured cells at nearly 4,400 frames per second-orders of magnitude faster than conventional imaging techniques, and with roughly equivalent spatial resolution. FIRE also performed well in flow cytometry with fluorescently labeled cells, collecting blurfree image data at a rate that greatly outpaced that of existing commercial instruments. “They're running at flow rates of a couple of centimeters per second, while we're run- ning at a few meters per second right now," says Diebold. He sees flow cytometry as a particularly promising early application, where FIRE's capacity for rapid data collection could prove invaluable in detecting rare circulating tumor cells in cancer patients. "It's looking for a needle in a haystack," he says, "but if you have an instrument that can give you throughput of 100,000 cells per second, you can find one in a billion cells in a matter of minutes." These remarkable frame rates could also offer critical insights into fastmoving cellular events such as neuronal signaling, and Jalali's group is now working with neurobiologists to develop a two-photon version of FIRE that might achieve equally highspeed imaging within the living brain.

\section{Michael Eisenstein}

RESEARCH PAPERS

Diebold, E.D. et al. Digitally synthesized beat

frequency multiplexing for sub-millisecond

fluorescence microscopy. Nat. Photonics 7, 806-810 (2013). 